\title{
Clinical Profile, Response to therapy and outcomes of post-ischemic intestinal stenosis Secondary to Acute Mesenteric Venous Thrombosis: a retrospective case series study
}

\section{Shilong Sun}

Nanjing University https://orcid.org/0000-0002-2943-6655

Xinyu Wang

Jinling Hospital

Chengnan Chu

Jinling Hospital

Zehua Duan

Jinling Hospital

Chao Yang

Jinling Hospital

Jiao Zong

Jinling Hospital

Baochen Liu

Jinling Hospital

Xingjiang Wu

Jinling Hospital

Qiurong Li

Jinling Hospital

Weiwei Ding ( $\nabla$ dingwei_nju@hotmail.com )

https://orcid.org/0000-0002-5026-689X

Research article

Keywords: Acute mesenteric venous thrombosis, Post-ischemic intestinal stenosis, Intestinal stricture, Hypoproteinemia, lleus, Surgical resection

Posted Date: June 25th, 2020

DOI: https://doi.org/10.21203/rs.3.rs-35279/v1 
License: (c) (i) This work is licensed under a Creative Commons Attribution 4.0 International License. Read Full License 


\section{Abstract \\ Background}

Acute mesenteric venous thrombosis (AMVT) can not only cause acute bowel infarction but result in post-ischemic intestinal stenosis (PIIS), which is rarely reported.

\section{Methods}

Consecutive patients diagnosed with PIIS secondary to AMVT (during the post-thrombotic course, confirmed segmental bowel stenosis) from January 2010 to June 2018 were enrolled. We evaluated the clinical profile, response to therapy, and outcomes of this complication managed at the intestinal stroke center in China. Clinical data are retrospectively analyzed and Wilcoxon signed-rank test was used to evaluate the efficiency of conservative treatment.

\section{Results}

42 patients with PIIS secondary to AMVT were evaluated. 20 (5-150) days after discharge for AMVT, symptoms of PIIS appeared, and the interval between symptom onset and consultation was 29.5 (2-180) days. In addition, abdominal pain (90.5\%), abdominal distension (76.2\%), weight loss $(71.4 \%)$, and nausea or vomiting (54.8\%) were the common presentations. Hypoproteinemia was observed in $61.9 \%$ of patients, while leukocytosis, anemia and electrolyte disorders were present in $11.9 \%, 45.2 \%$ and $35.7 \%$ of patients, respectively. CT excluded thrombosis recurrence and revealed extensive collateral circulation or portal cavernous transformation in $83.3 \%$ of patients. Enterography was required only in $81.0 \%$ of patients, with positive findings in all patients, including complete obstruction (23.5\%), intestinal stricture (64.7\%), and extensive mucosal erosion (11.8\%). Prompt conservative treatment achieved significantly increases of albumin and pre-albumin levels and correction of electrolyte disorders (all $p<0.05$ ). However, only $4(9.5 \%)$ patients improved after conservative therapy and laparotomy was required in other patients. A median length of 20 (2-100) cm of involved bowel was resected, mainly involving the jejunum (76.3\%). Only 1 patient died due to uncontrolled transverse colon bleeding, and other patients' postoperative courses were uneventful.

\section{Conclusion}

During the post-thrombotic course, clinicians should be aware of the possibility of PIIS in patients with symptoms of ileus and hypoproteinemia. Patients may respond to conservative therapy; however, the majority of patients warrant surgical resection.

\section{Background}


Acute mesenteric venous thrombosis (AMVT) accounts for 1 in 1000 emergency department admissions and $6-28 \%$ of cases of acute mesenteric ischemia[1,2]. AMVT can cause lethal outcomes, and the mortality is up to $20 \%$ [3]. The natural history and long-term sequelae of AMVT are little known[4]. In the short term, AMVT can cause intestinal infarction, which is the main cause of poor prognosis, and secondary short bowel syndrome and recurrent thrombosis can also affect outcomes[1]. In the long term, secondary portal hypertension and portal cholangiopathy, which mostly occur in the chronic phase, are also considered chronic complications[4, 5].

However, the chronic complication of delayed intestinal stricture secondary to AMVT, post-ischemic intestinal stenosis (PIIS), has rarely been reported[6, 7]. In 1985, Witte et al first reported the onset of mesenteric and portal vein thrombosis in a 71-year-old man, and the barium follow-up showed edema, spicules, thumb printing and poor distensibility of the distal ileum[8]. Since then, only a few case reports have been found. Given the rare nature of PIIS secondary to AMVT, most articles on this condition have been isolated case reports or case series[9]. Systemic studies of the characteristics are scanty, especially in terms of follow-up and long-term outcomes. We evaluated the clinical profile, response to therapy, complications and outcomes of PIIS managed at our intestinal stroke center (ISC).

\section{Materials And Methods}

\section{Patient characteristics}

This case seires research was conducted in an integrated ISC in China, which was set up in 2010[10, 11]. The study was approved by the Institutional Review Board of Jinling Hospital. Informed consent was obtained from each patient and their relatives. 42 consecutive patients diagnosed with PIIS secondary to AMVT from January 2010 to June 2018 in the ISC were evaluated. The diagnosis of PIIS secondary to AMVT was based on the following criteria: (1) patients in post-thrombotic course with a treatment history of AMVT and/or with extensive collateral circulation (or portal cavernous transformation) in mesenteric venules[5]; and (2) patients with segmental bowel stenosis, which was confirmed by radiologic findings and/or laparotomy [12,13] (Fig. 1).

Detailed information regarding the demographics, clinical manifestations, and findings on general physical and systemic examinations was recorded. All patients were evaluated by complete hemograms and liver function tests, etc. In addition, all patients underwent contrast-enhanced computed tomography (CT) to evaluate the venous drainage of the portal venous system, and nonvascular findings can help assess intestinal damage[2]. Furthermore, enterography with gastrografin was performed to evaluate intestinal patency and mucosal patterns in the ISC[13-15] (Briefly, gastrografin was administered through a naso-intestinal tube, and serial images of the transit of gastrografin was obtained by abdominal radiography to evaluate the ability of intestinal peristalsis and patency. Complete obstruction or appearance of contrast in the ascending colon was the ending criterion. In addition, the naso-intestinal tube was used for decompression after the test, with a strict measurement of output being performed.). 


\section{Management And Follow-up}

All patients were managed with prompt supportive therapy, including anticoagulation, correction of electrolyte disorders, etc. The nutrition support modality was under the guidance of a clinical nutrition expert[11]. Planned/emergency laparotomy was indicated to resect the involved small intestine by two experienced surgeons when general conditions could not be corrected or when symptom deterioration occurred[9]. The postoperative nutrition support modality was started gradually. In addition, lowmolecular-weight heparin calcium or oral anticoagulants were administered after the exclusion of risk factors for bleeding[5].

Follow-up surveys were conducted in the outpatient clinic at 1 and 6 months and every 12 months thereafter. Radiologic findings and clinical manifestations, complications, mortality, and morbidity were obtained to assess treatment outcomes.

\section{Data analysis}

Data are presented as medians with ranges and proportions. The Wilcoxon signed-rank test was used to compare two related samples. A p value of $<0.05$ was considered significant. Statistical analysis was performed using SPSS software for Windows version 23.0 (SPSS, Chicago, IL, USA).

\section{Results}

\section{Demographics and Clinical Features}

42 patients with PIIS secondary to AMVT (29 (69.0\%) males, age at diagnosis of 50 (25-66) years) were evaluated. The patients' characteristics and risk factors are described in Table 1. In these patients, the risk factors for thrombosis included splenectomy $(n=6,14.3 \%)$, protein C/S deficiency $(n=5,11.9 \%)$ and antithrombin III deficiency $(n=4,9.5 \%)$. Among these patients, 16 (38.1\%) underwent mesenteric revascularization after systemic anticoagulation alone, while the other $26(61.9 \%)$ patients were managed with prompt endovascular treatment in the acute phase of AMVT. However, approximately 20 (5-150) days after discharge for AMVT, symptoms of PIIS appeared, and the interval between symptom onset and consultation was 29.5 (2-180) days. In addition, abdominal pain ( $n=38,90.5 \%)$, abdominal distension $(n=32,76.2 \%)$, weight loss $(n=30,71.4 \%)$, nausea or vomiting $(n=23,54.8 \%)$, and postprandial pain $(n=22$, $52.4 \%$ ) were the main clinical manifestations (Table 2).

Hypoproteinemia ( $<60 \mathrm{~g} / \mathrm{L}$ ) and hypoalbuminemia ( $<35 \mathrm{~g} / \mathrm{L})$ were each observed in $26(61.9 \%)$ patients, while leukocytosis $\left(>10 \times 10^{\wedge} 9 / \mathrm{L}\right)$ and leukopenia $\left(<4 \times 10^{\wedge} 9 / \mathrm{L}\right)$ were present in $5(11.9 \%)$ and $7(16.7 \%)$ patients, respectively. Moreover, anemia (hemoglobin $<110 \mathrm{~g} / \mathrm{L}$ ) and electrolyte disorders $(\mathrm{K}+<3.5 \mathrm{mmol} / \mathrm{L}$ or $\mathrm{Na}+<135 \mathrm{mmol} / \mathrm{L})$ were found in $19(45.2 \%)$ and 15 (35.7\%) patients, respectively. CT was carried out in all patients, and the recurrence of thrombosis in mesenteric veins was excluded. The vascular findings from the CT scan included extensive collateral circulation $(n=18,42.9 \%)$, portal cavernous transformation $(n=17,40.5 \%)$, gastric varices $(n=11,26.2 \%)$, and lack of luminal enhancement $(n=10,23.8 \%)$. The main 
nonvascular findings included regional bowel wall thickening (target sign) $(n=13,31.0 \%)$ and bowel dilatation ( $n=23,54.8 \%)$. Enterography was required in $34(81.0 \%)$ patients and revealed different degrees of intestinal stenosis: intestinal stricture $(n=22,64.7 \%)$, dilation of the proximal intestinal segments $(n=18,52.9 \%)$, and extensive mucosal erosion ( $n=4,11.8 \%$ ) (Figure 2). Moreover, complete obstruction with severe dilation of the proximal intestinal segments were found in $8(23.5 \%)$ patients. This test also revealed that the jejunum was mainly involved in $24(70.6 \%)$ patients, while the other $10(29.4 \%)$ patients suffered from ileal stenosis.

\section{Response to Therapy and outcome}

Prompt conservative treatments were initiated upon diagnosis. In addition to systemic anticoagulation and supportive therapy, all patients were required to undergo nutrition support for approximately 10 (5-30) days $(11(26.2 \%)$ patients with enteral nutrition through naso-intestinal tubes and 31 (73.8\%) patients with parenteral nutrition because of complete ileus or intolerance to enteral nutrition), and 14 (33.3\%) patients were given albumin infusion. Significantly increases of albumin and pre-albumin levels and correction of electrolyte disorders were found after conservative treatment (all $p<0.05$ ). The comparison of anthropometry and laboratory profiles before and after initial supportive therapy is shown in Table 3 .

However, only 4 (9.5\%) patients achieved symptom alleviation and were discharged later. Surprisingly, despite prompt nutritional therapy, 23 (54.8\%) patients still suffered from constant hypoproteinemia, and decreased total protein levels were also found in 12 (28.6\%) patients. In addition, 5 (11.9\%) patients experienced symptom deterioration during nutrition support, accompanied by elevated leukocyte counts. Notably, repeated enterography was required in these 5 patients, and aggravated intestinal stenosis was found after comparison (Figure 3).

Eventually, laparotomy was required in 38 out of 42 patients (31 (81.6\%) patients with planned laparotomies and 7 (18.4\%) patients with emergency laparotomies), and a median length of a 20 (2-100)$\mathrm{cm}$-long involved section of the small intestine was resected (Table 4). The jejunum was mainly involved in $29(76.3 \%)$ patients, while the other $9(23.7 \%)$ patients suffered from ileal stenosis. The common findings of histological examinations revealed ischemic injury, including chronic inflammation and surrounding granulation tissue formation with ulceration or fibrosis in submucosal layers (Figure $1 \mathrm{~d}$ ). In addition to supportive therapy, postoperative nutrition therapy was initiated for approximately 10 (4-29) days, and anticoagulation was also administered after recurrence of thrombosis and after the exclusion of bleeding. All patients achieved symptom alleviation, and hypoproteinemia was corrected after surgical resection.

\section{Complications and follow-up}

One patient died due to severe digestive tract bleeding in the transverse colon. Intra-abdominal sepsis occurred in three patients. One patient had anastomotic leakage and was cured after conservative treatment. Moreover, two patients died as a result of malignancy, three patients required splenectomy, and four patients underwent transjugular intrahepatic portosystemic shunting because of secondary portal 
hypertension during follow-up. The other patients' postoperative courses were uneventful, and follow-up examinations revealed an unremarkable state of health. The 30-day and one-year mortality rates were $2.4 \%$ and $7.1 \%$, respectively.

\section{Discussion}

Since 2010, more than 158 patients with acute mesenteric ischemia have been administered in the ISC by a multidisciplinary stepwise management strategy, and a better outcome was achieved, as shown in previous reports[10, 16, 17]. However, in clinical practice and follow-up, we found that many patients with AMVT suffered from segmental intestinal stenosis in the long term, even though those patients successfully underwent mesenteric revascularization in the acute phase. Surprisingly, stenosis of the small bowel is rarely mentioned in reports of mesenteric venous thrombosis, suggesting that it may be a rare complication[18]. In this large study, we evaluated 42 patients with PIIS secondary to AMVT and showed that the triad of abdominal pain, abdominal distention and vomiting was present in $55 \%$ of patients. In addition, hypoproteinemia and hypoalbuminemia were present in $62 \%$ of patients.

Surprisingly, hypoproteinemia could not be corrected with prompt initial nutrition support and symptom alleviation can't be achieved after conservative therapy in most cases. Ultimately, resection of the involved segmental intestine and postoperative nutrition resulted in a good general outcome, and the postoperative courses were uneventful in most patients.

Signs appear during the post-thrombotic course, and the main triad of symptoms, which indicates the appearance of chronic intestinal obstruction as a result of intestinal stenosis, mainly occurs several weeks after revascularization (it varies from several days to months)[6]. Ischemic intestinal stenosis does meet the diagnostic criteria of ischemic enteritis, which can also be related to limited thrombosis or embolism[19, 20]. However, in this research, we found that constant malnutrition with hypoproteinemia and hypoalbuminemia was another main clinical feature. In addition, Matsushita et al also demonstrated that it is a cause of protein-losing enteropathy (PLE) with the use of technetium 99 m-labeled human serum albumin scintigraphy[21]. However, postprandial pain and intolerance to oral intake may be another cause of malnutrition. Thus, the term "postischemic intestinal stenosis" instead of "ischemic enteritis" was used in this research.

CT has been useful for the diagnosis of AMVT, as it produces a specificity of $90 \%$ and a sensitivity of $95 \%[5,22]$. It can evaluate the venous drainage of the portal venous system and exclude the possibility of thrombosis recurrence and the nonvascular findings can help assess intestinal ischemia. Segmental bowel wall thickening and bowel dilation are the main nonvascular findings, which indicates the appearance of bowel edema and the possibility of intestinal obstruction. Moreover, extensive collaterals are also found in most cases. Prompt revascularization and the development of sufficient collaterals ensure venous drainage from the involved ischemic bowels[23]. However, in some cases, the collaterals might not be adequate for preventing segmental chronic bowel ischemia, which has been demonstrated in the histological examination of chronic inflammation in most resected specimens. A similar result was also found by Eugene et a/ who reported that intestinal strictures associated with mesenteric vein 
thrombosis were probably the consequence of ischemia[6]. Interestingly, involvement of the colon has not yet been found, and the reason for this may be better collateralization of the colonic venous system, preventing chronic ischemia and therefore ischemic stenosis[13].

Barium examination is considered to be most useful for the diagnosis of ischemic enteritis[14]. However, barium studies may be dangerous in cases of nearly complete obstruction, as it may become inspissated above the level of obstruction, or barium may spread into the peritoneal cavity if perforation occurs or is present[24]. In this research, complete obstruction was found in 8 patients, and severe stenosis was also found in many patients. By contrast, gastrografin, a water-soluble contrast medium, can decrease edema of the bowel wall and enhance bowel motility, and it can be easily diluted by enteric fluid and can enhance mucosal details on radiography[25]. Furthermore, water-soluble contrast medium of high osmolarity can accelerate the resolution of postoperative ileus and enhance the resolution of adhesive bowel obstruction[24, 25]. Positive findings of intestinal stenosis were found in all patients who underwent this test. The location of the involved intestine, which varies from the jejunum to the ileum, can be identified by the use of gastrografin, which has a beneficial effect on subsequent surgical interventions.

Stricture in ischemic enteritis sometimes tends to progressively worsen and ultimately results in total occlusion[15,26]. Similar results may also occur in PIIS secondary to AMVT. The condition of 5 patients worsened from mild symptoms to severe abdominal pain during initial nutrition therapy. Moreover, the worsen of intestinal stenosis and the increase in leukocyte counts may also indicate the aggravation of intestinal ischemia and the initiation of a systemic inflammatory response[16, 27]. According to the comparison of enterography findings in these patients, we hypothesize that "mucosal erosion-stricturecomplete obstruction" may also be the progression of PIIS secondary to AMVT to some extent. Thus, in the 4 patients who experienced symptom alleviation and were discharged after conservative treatment, close follow-up after discharge is still warranted and has already been carried out.

90.5\% patients do not improve with conservative treatment and nutritional therapy. In contrast, postoperative nutrition therapy can effectively correct constant hypoproteinemia, and symptoms can improve after resection of the involved intestines. The postoperative course was uneventful in most patients. Thus, as patients may improve promptly with conservative management, it is better to perform surgery, and prompt surgical resection may lead to better outcomes. Surgical resection is also the main strategy of intestinal enteritis[20]. An emerging role of balloon dilation for symptomatic intestinal stricture has also been reported and is considered to be a useful alternative to surgery when the length of the stricture is less than $3 \mathrm{~cm}[19]$. However, small bowel strictures that are long $(>3 \mathrm{~cm})$ may not be candidates for balloon dilation, and the length of the involved intestine in PIIS secondary to AMVT was longer than $3 \mathrm{~cm}$ in most patients. Moreover, endoscopic balloon dilation of strictures carries a risk of perforation at rates of up to 2.9\%[28]. Therefore, the role of balloon dilation in PIIS secondary to AMVT should be further evaluated and warrants additional research in the future. 
The main limitations of the study include its retrospective nature and the fact that the clinical data were limited in the records. Moreover, the diagnostic effect of endoscopy, especially capsule endoscopy, was not discussed, which allows for precise examination of the small intestine[26], and it could not be done due to non-availability in the research period. However, a prospective study is already in progress.

\section{Conclusions}

In conclusion, PIIS is an uncommon but important long-term complication of AMVT. During the postthrombotic course, clinicians should be aware of the possibility of PIIS in patients with symptoms of ileus and hypoproteinemia. Patients may respond to conservative therapy; however, the majority of patients warrant surgical resection. Early recognition and prompt treatment may lead to a better outcome.

\section{Abbreviations}

AMVT

Acute mesenteric venous thrombosis

PIIS

post-ischemic intestinal stenosis

ISC

intestinal stroke center

CT

computed tomography

\section{Declarations}

\section{Availability of data and materials}

The datasets used and/or analysed during the current study are available from the corresponding author upon reasonable request.

\section{Acknowledgements:}

Not applicable.

\section{Funding:}

This work was funded by the National Natural Science Foundation of China (81770532) and Jiangsu Province Medical Foundation for Youth Talents (QNRC2016901).

\section{Author information:}

Shilong Sun and Xinyu Wang contributed equally to this work. 


\section{Affiliations}

Division of Trauma and Surgical Intensive Care Unit, Department of Critical Care Medicine, Research Institute of General Surgery, Jinling Hospital, Medical School of Nanjing University, Nanjing 210002, Jiangsu Province, P.R. China.

Shilong Sun, Xinyu Wang, Chengnan Chu, Zehua Duan, Jiao Zong, Chao Yang, Kai Wang, Baochen Liu, Xingjiang Wu, Qiurong Li \& Weiwei Ding

Integrated Intestinal Stroke Center, Research Institute of General Surgery, Jinling Hospital, Medical School of Nanjing University, Nanjing 210002, Jiangsu Province, P.R. China.

Shilong Sun, Xinyu Wang, Chengnan Chu, Zehua Duan, Jiao Zong, Chao Yang, Kai Wang, Baochen Liu, Xingjiang Wu, Qiurong Li \& Weiwei Ding

\section{Contributions}

SLS and XYW conceived and written the study; CNC, ZHD and JZ carried out the data collection; CY and KW followed up patients; BCL and XJW conducted the data analysis. QRL and WWD revised the paper; SLS and XYW are co-first authors. All authors have read and approved the final version of this manuscript.

\section{Corresponding author}

Corresponding to Weiwei Ding and Qiurong Li.

\section{Ethic declarations}

\section{Ethics approval and consent to participate}

This study was conducted in accordance with the declaration of Helsinki. Informed consent from the patients was waived because of the retrospective nature of this study, and this study was approved by Institutional Ethnic Committee of the Jinling Hospital (2018NZGKJ-019)

\section{Consent for publication}

The written consents for the publication of the images were obtained from the patients. The completed written versions are available in the Patients' Medical History (Research Institute of General Surgery, Jinling Hospital, Medical School of Nanjing University).

\section{Competing interests:}

All authors declare no conflicts of interest.

\section{References}


1. Singal AK, Kamath PS, Tefferi A. Mesenteric venous thrombosis. Mayo Clin Proc. 2013;88(3):285 94.

2. Bjorck M, Koelemay M, Acosta S, Bastos Goncalves F, Kolbel T, Kolkman JJ et al Editor's Choice Management of the Diseases of Mesenteric Arteries and Veins: Clinical Practice Guidelines of the European Society of Vascular Surgery (ESVS). Eur J Vasc Endovasc Surg. 2017;53(4):460-510.

3. Acosta S, Alhadad A, Svensson P, Ekberg O. Epidemiology, risk and prognostic factors in mesenteric venous thrombosis. Br J Surg. 2008;95(10):1245-51.

4. Maldonado TS, Blumberg SN, Sheth SU, Perreault G, Sadek M, Berland T, et al. Mesenteric vein thrombosis can be safely treated with anticoagulation but is associated with significant sequelae of portal hypertension. J Vasc Surg Venous Lymphat Disord. 2016;4(4):400-6.

5. Harnik IG, Brandt LJ. Mesenteric venous thrombosis. Vasc Med. 2010;15(5):407-18.

6. Eugene C, Valla D, Wesenfelder L, Fingerhut A, Bergue A, Merrer J, et al. Small intestinal stricture complicating superior mesenteric vein thrombosis. A study of three cases. Gut. 1995;37(2):292-5.

7. Elkrief L, Corcos O, Bruno O, Larroque B, Rautou PE, Zekrini $K$, et al. Type 2 diabetes mellitus as a risk factor for intestinal resection in patients with superior mesenteric vein thrombosis. Liver Int. 2014;34(9):1314-21.

8. Witte CL, Brewer ML, Witte MH, Pond GB. Protean manifestations of pylethrombosis. A review of thirty-four patients. Ann Surg. 1985;202(2):191-202.

9. Paraskeva P, Akoh JA. Small bowel stricture as a late sequela of superior mesenteric vein thrombosis. Int J Surg Case Rep. 2015;6C:118-21.

10. Yang S, Fan X, Ding W, Liu B, Meng J, Xu D, et al. Multidisciplinary stepwise management strategy for acute superior mesenteric venous thrombosis: an intestinal stroke center experience. Thromb Res. 2015;135(1):36-45.

11. Ding W, Wang K, Liu B, Fan X, Wang S, Cao J, et al. Open Abdomen Improves Survival in Patients With Peritonitis Secondary to Acute Superior Mesenteric Artery Occlusion. J Clin Gastroenterol. 2017;51(9):e77-82.

12. Arentsen JC, van den Anker-Lugtenburg PJ, Jonkers GH, Schipper ME, Michiels JJ, van Buuren HR. Short-segment jejunal stenosis complicating subacute portomesenteric venous thrombosis in a patient with protein S deficiency type II. Am J Gastroenterol. 1996;91(8):1653-4.

13. Yang J, Shen L, Zheng X, Zhu Y, Liu Z. Small bowel stricture complicating superior mesenteric vein thrombosis. J Huazhong Univ Sci Technolog Med Sci. 2012;32(1):146-8.

14. lida M, Matsui T, Yao T, Iwashita A, Fujishima M. Radiographic features in ischemic jejunoileitis: serial changes and comparison with pathologic findings. Gastrointestinal Radiology. 1992;17(4):327-32.

15. Takeuchi N, Naba K. Small intestinal obstruction resulting from ischemic enteritis: a case report. Clin J Gastroenterol. 2013;6(4):281-6. 
16. Wang X, Chu C, Sun S, Xie T, Duan Z, Wang K, et al. Outcomes and clinical characteristics of transmural intestinal necrosis in acute mesenteric ischemia. Scand J Gastroenterol. 2019;54(8):9539.

17. Liu K, Meng J, Yang S, Liu B, Ding W, Wu X, et al. Transcatheter thrombolysis combined with damage control surgery for treatment of acute mesenteric venous thrombosis associated with bowel necrosis: a retrospective study. World J Emerg Surg. 2015;10:50.

18. Kim HK, Chun JM, Huh S. Anticoagulation and delayed bowel resection in the management of mesenteric venous thrombosis. World J Gastroenterol. 2013;19(30):5025-8.

19. Nishimura N, Yamamoto H, Yano T, Hayashi Y, Sato H, Miura Y, et al. Balloon dilation when using double-balloon enteroscopy for small-bowel strictures associated with ischemic enteritis. Gastrointest Endosc. 2011;74(5):1157-61.

20. Koshikawa $Y$, Nakase $H$, Matsuura M, Yoshino T, Honzawa Y, Minami N, et al. Ischemic enteritis with intestinal stenosis. Intest Res. 2016;14(1):89-95.

21. Matsushita I, Hanai H, Sato Y, Arai H, lida T, Hosoda Y, et al. Protein-losing enteropathy caused by mesenteric venous thrombosis with protein C deficiency. J Clin Gastroenterol. 2000;30(1):94-7.

22. Acosta S, Alhadad A, Ekberg O. Findings in multi-detector row CT with portal phase enhancement in patients with mesenteric venous thrombosis. Emerg Radiol. 2009;16(6):477-82.

23. Grendell JH, Ockner RK. Mesenteric venous thrombosis. Gastroenterology. 1982;82(2):358-72.

24. Chen SC, Lin FY, Lee PH, Yu SC, Chang KJ. Water-soluble contrast study predicts the need for early surgery in adhesive small bowel obstruction. Br J Surg. 1999;85(12):1692-4.

25. Choi H, Chu K, Law W. Therapeutic value of Gastrografin in adhesive small bowel obstruction after unsuccessful conservative treatment: A prospective randomized trial. Ann Surg. 2002;236(1):1-6.

26. Yang XY, Chen CX, Zhang BL, Yang LP, Li YM. Diagnostic effect of capsule endoscopy in 31 cases of subacute small bowel obstruction. World J Gastroenterol. 2009;15(19):2401-5.

27. Emile SH. Predictive Factors for Intestinal Transmural Necrosis in Patients with Acute Mesenteric Ischemia. World J Surg. 2018;42(8):2364-72.

28. Mensink PBF. Complications of Double Balloon Enteroscopy. Techniques in Gastrointestinal Endoscopy.10(2):0-69.

\section{Tables}




\begin{tabular}{|c|c|}
\hline Clinical Features & PIIS secondary to AMVT $(n=42)$ \\
\hline Age, y & $50(25-66)$ \\
\hline \multicolumn{2}{|l|}{ Gender } \\
\hline Male & $29(69.0)$ \\
\hline Female & $13(31.0)$ \\
\hline \multicolumn{2}{|l|}{ Etiology } \\
\hline \multicolumn{2}{|l|}{ Secondary thrombophilia } \\
\hline Splenectomy & $6(14.3)$ \\
\hline Thrombocythemia & $3(7.1)$ \\
\hline Cirrhosis & $3(7.1)$ \\
\hline Inflammatory bowel disease & $1(2.4)$ \\
\hline Pancreatitis & $2(4.8)$ \\
\hline Appendectomy & $2(4.8)$ \\
\hline Other abdominal surgery & $2(4.8)$ \\
\hline Pregnancy & $2(4.8)$ \\
\hline \multicolumn{2}{|l|}{ Inherited thrombophilia } \\
\hline Protein C/S deficiency & $5(11.9)$ \\
\hline Anti-thrombin III deficiency & $4(9.5)$ \\
\hline \multicolumn{2}{|l|}{ Comorbidity } \\
\hline Hypertension & $6(14.3)$ \\
\hline Portal hypertension & $8(19.0)$ \\
\hline Stroke & $1(2.4)$ \\
\hline Prior deep vein thrombosis & $9(21.4)$ \\
\hline Vasculitis & $1(2.4)$ \\
\hline Smoking & $6(14.3)$ \\
\hline Alcohol abuse & $6(14.3)$ \\
\hline
\end{tabular}


*All values are expressed as the median and range or $n(\%)$. PIIS: Post-ischemic intestinal stenosis. AMVT: Acute mesenteric venous thrombosis. 
Table 2

Clinical presentations and imaging characteristics of patients with PIIS secondary to AMVT.

Clinical Features

PIIS secondary to AMVT $(n=42)$

Clinical presentation

Abdominal pain

$38(90.5)$

Abdominal distension

$32(76.2)$

Nausea or vomiting

$23(54.8)$

Constipation

$16(38.1)$

Weight loss

30 (71.4)

Postprandial pain

$22(52.4)$

Fever

2 (4.8)

Duration from symptom onset to consultation, $d$

$29.5(2-180)$

\section{Characteristics of CT imaging}

Vascular findings

Lack of luminal enhancement

$10(23.8)$

Extensive collateral circulation

18 (42.9)

Portal cavernous transformation

17 (40.5)

Gastric varices

$11(26.2)$

\section{Nonvascular findings}

Bowel wall thickening

$13(31.0)$

Bowel dilatation

$23(54.8)$

Mesenteric fat stranding

$2(4.8)$

Splenomegaly

4 (9.5)

Ascites

$2(4.8)$

\section{Enterography $(n=34)$}

Mucosal erosion

Intestinal stenosis

$22(64.7)$

Severe dilation of the proximal intestine

$18(52.9)$

Complete obstruction

$8(23.5)$ 
*All values are expressed as the median and range or $\mathrm{n}(\%)$. PIIS: Post-ischemic intestinal stenosis. AMVT: Acute mesenteric venous thrombosis. CT: Computed tomography.

\section{Table 3: Comparison of the laboratory profiles of patients before and after prompt supportive conservative therapy}

\begin{tabular}{|c|c|c|c|}
\hline Laboratory parameters & Upon admission & After therapy & $P$ value \\
\hline Body mass index, $\mathrm{kg} / \mathrm{m}^{\wedge} 2$ & $19.9(15.2-24.2)$ & $20.0(15.6-22.9)$ & 0.334 \\
\hline Leukocyte count, $\times 10^{\wedge} 9 / \mathrm{L}$ & $5.3(1.4-29.0)$ & $5.2(2.9-16.7)$ & 0.741 \\
\hline C-reactive protein, $\mathrm{mg} / \mathrm{L}$ & $11.5(2.0-98.1)$ & $18.2(0.6-198.0)$ & 0.478 \\
\hline Procalcitonin, $\mu \mathrm{g} / \mathrm{L}$ & $0.18(0.02-15.0)$ & $0.18(0.02-3.60)$ & 0.469 \\
\hline Hemoglobin, $g / L$ & $109.0(53.0-137.0)$ & $100.5(80.0-191.0)$ & 0.886 \\
\hline Platelet count, $\times 10^{\wedge} 9 / \mathrm{L}$ & $199.0(26.0-597.0)$ & $157.0(13.0-576.0)$ & 0.798 \\
\hline Total protein, $\mathrm{g} / \mathrm{L}$ & $56.0(37.0-81.0)$ & $59.2(42.0-77.0)$ & 0.080 \\
\hline Albumin, g/L & $33.0(16.0-44.0)$ & $36.4(23.0-53.0)$ & 0.021 \\
\hline Pre-albumin, g/L & $100.0(10.0-239.0)$ & $110.0(60.0-220.0)$ & 0.033 \\
\hline Alanine aminotransferase, $\mathrm{U} / \mathrm{L}$ & $22.0(4.0-113.0)$ & $21.0(7.0-63.0)$ & 0.340 \\
\hline $\mathrm{Na}^{+}$ & $136.5(124.0-145.0)$ & $138.0(134.0-147.0)$ & 0.038 \\
\hline $\mathrm{K}^{+}$ & $4.0(1.9-5.3)$ & $4.1(3.6-5.3)$ & 0.019 \\
\hline \multicolumn{4}{|c|}{$\begin{array}{l}\text { *All values are expressed as the median and range or } \mathrm{n}(\%) \text {. PIIS: Post-ischemic intestinal stenosis. } \\
\text { AMVT: Acute mesenteric venous thrombosis. Normal range for body mass index: } 18.5-23.9 \mathrm{~kg} / \mathrm{m}^{\wedge} 2 \text {; } \\
\text { Normal range for leukocyte count: } 4-10 \times 10^{\wedge} 9 / \mathrm{L} ; \text { Normal range for C-reactive protein: } 0-8 \mathrm{mg} / \mathrm{L} ; \\
\text { Normal range for procalcitonin: } 0-0.5 \mu \mathrm{g} / \mathrm{L} \text {; Normal range for hemoglobin: } 110-160 \mathrm{~g} / \mathrm{L} ; \text { Normal range } \\
\text { for platelet count: } 100-300 \times 10^{\wedge} 9 / \mathrm{L} ; \text { Normal range for total protein: } 65-80 \mathrm{~g} / \mathrm{L} ; \text { Normal range for } \\
\text { albumin: } 35-50 \mathrm{~g} / \mathrm{L} ; \text { Normal range for pre-albumin: } 200-400 \mathrm{~g} / \mathrm{L} ; \text { Normal range for alanine } \\
\text { aminotransferase: } 0-38 \mathrm{U} / \mathrm{L} ; \text { Normal range for } \mathrm{Na}^{+}: 135-145 \mathrm{mmol} / \mathrm{L} \text {; Normal range for } \mathrm{K}+\llbracket 3.5-5.5 \\
\mathrm{mmol} / \mathrm{L} \text {. }\end{array}$} \\
\hline
\end{tabular}




\section{Table 4}

\section{Treatments, complications and follow-up outcomes of patients with PIIS secondary to AMVT.}

Clinical Features

PIIS secondary to AMVT $(n=42)$

Initial nutrition support

Enteral nutrition

$11(26.2)$

Parenteral nutrition

$31(73.8)$

Duration, d

$10(5-30)$

Albumin infusion

$14(33.3)$

Treatment strategies

Conservative treatment

$4(9.5)$

Bowel resection

38 (90.5)

Length of bowel resection, $\mathrm{cm}$

$20(2-100)$

Location of involved intestine

Jejunum

$29(76.3)$

Ileum

$9(23.7)$

Duration of postoperative nutrition support, $d$

$10(4-29)$

30-day mortality, \%

2.4

1-year mortality, \%

7.1

Major complications

Intra-abdominal sepsis

$3(7.1)$

Anastomotic leakage

1 (2.4)

Gastrointestinal bleeding

$1(2.4)$

Follow-up finding

Malignance

$2(4.8)$

Splenectomy

$3(7.1)$

TIPS

$4(9.5)$

*All values are expressed as the median and range or $\mathrm{n}(\%)$. PIIS: Post-ischemic intestinal stenosis. AMVT: Acute mesenteric venous thrombosis. TIPS: trans-jugular intrahepatic portosystemic shunt.

\section{Figures}




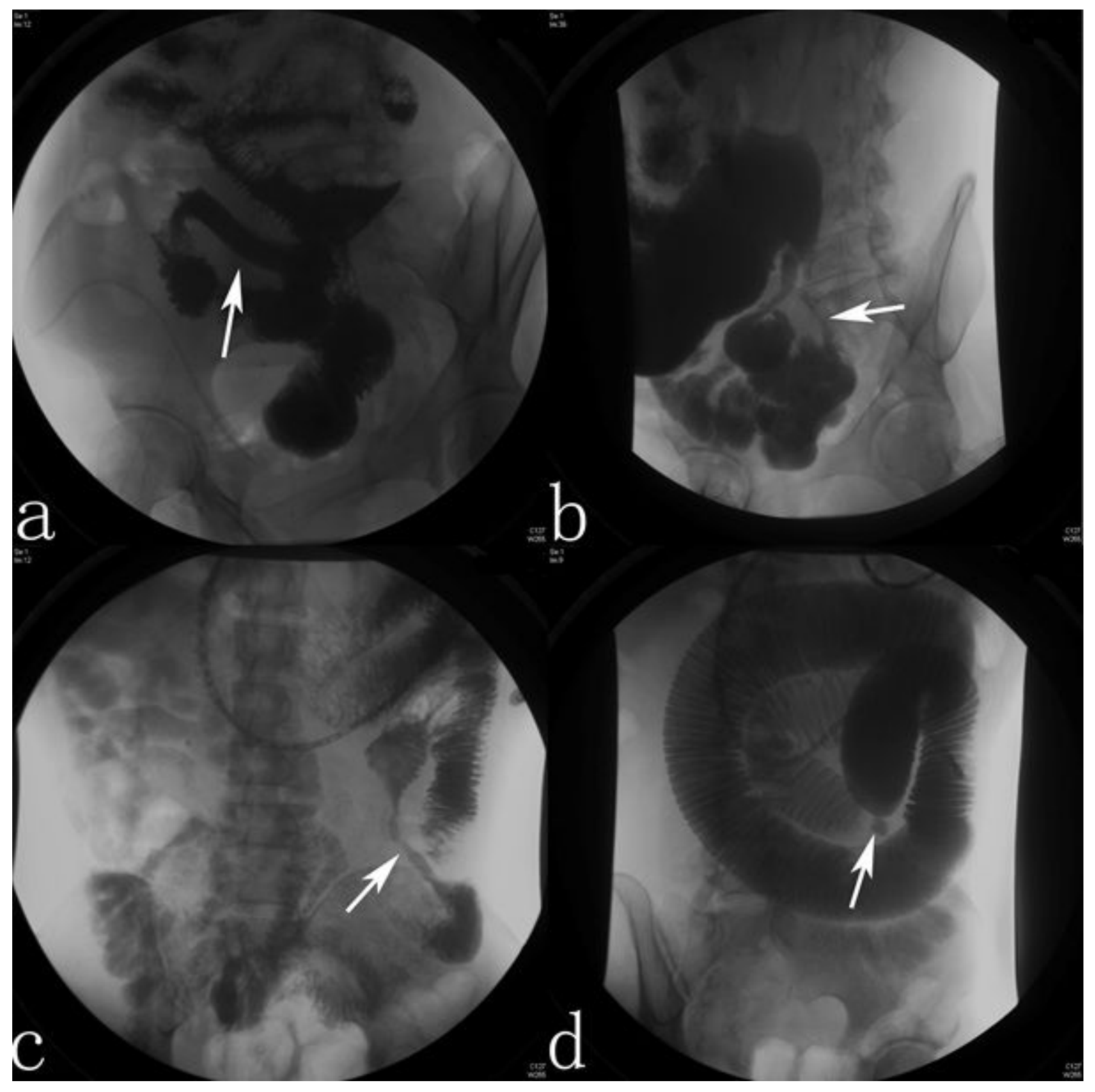

\section{Figure 1}

Comparison of enterography findings in two patients with symptom deterioration during nutritional support. a \& b: first enterography of a 26 -year-old patient revealed extensive mucosal erosion (a, arrow), and repeated testing of the same patient approximately 20 days later indicated severe bowel stenosis (arrow) with dilation of the proximal intestinal segments (b). Similarly, $c$ and d. c: enterography of a 44year-old man revealed segmental intestinal stricture, and a repeated test was carried out 25 days later, demonstrating complete obstruction. 


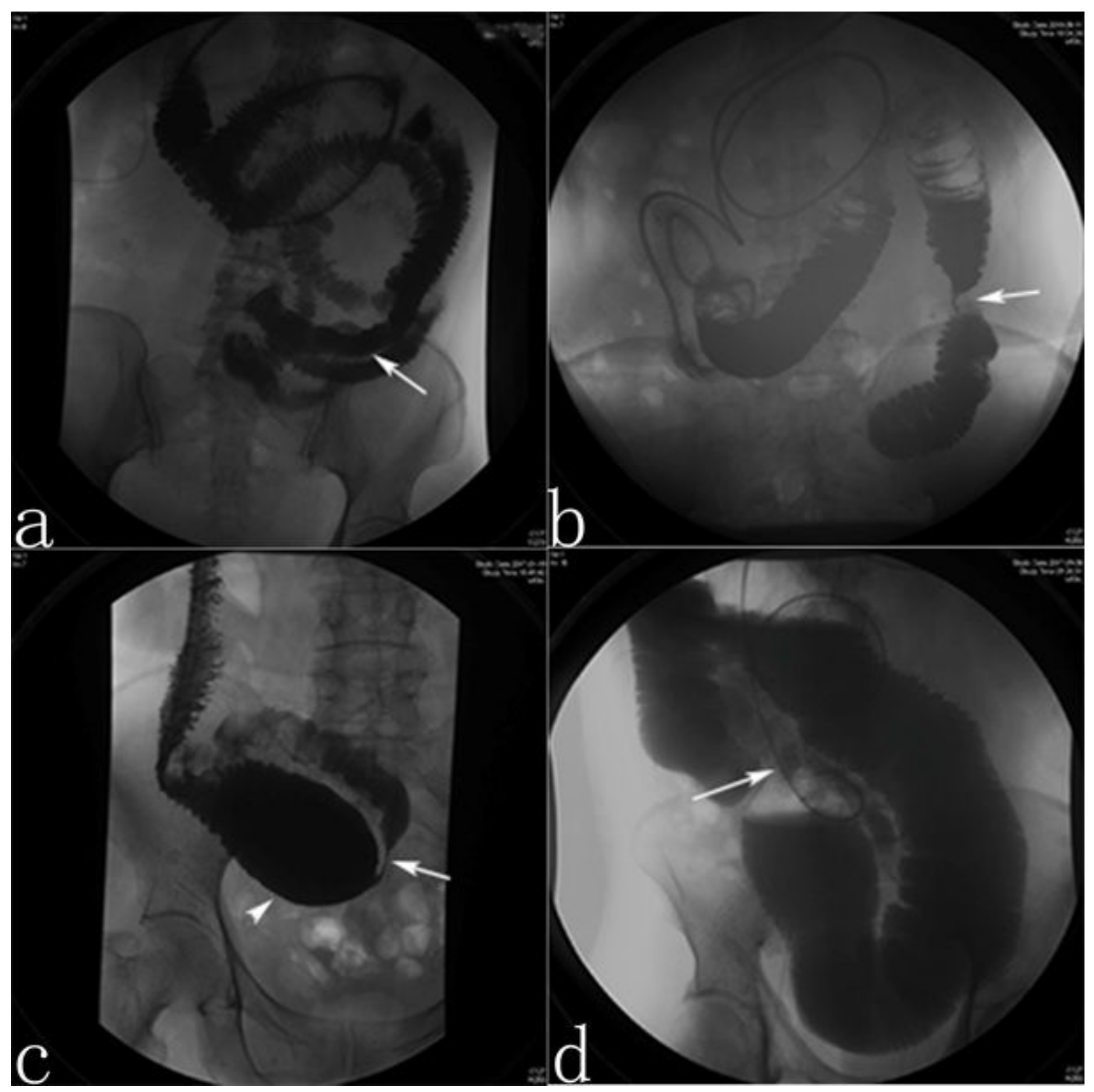

Figure 2

Different findings from enterography. a: extensive mucosal erosion/absence (white arrow). b: segmental bowel stricture (white arrow). c: severe dilation of the proximal intestinal segments (arrowhead) and segmental bowel stricture (white arrow). d: complete obstruction (white arrow). 


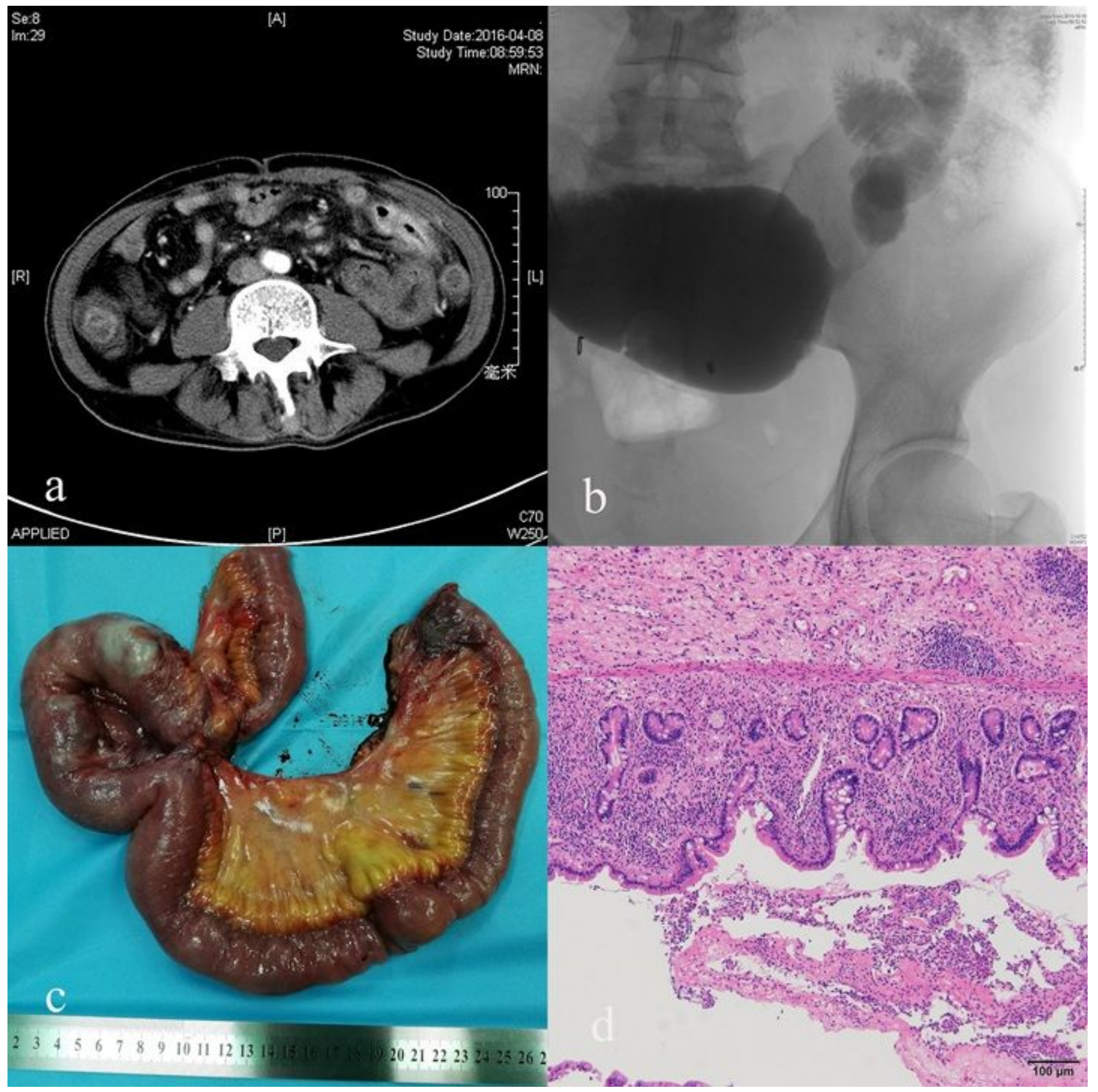

\section{Figure 3}

a: Computed tomography revealed segmental stenosis and bowel wall thickening (arrow); b:

Enterography revealed segmental bowel stricture (arrow); c: Dilated proximal jejunum and a 15-cm segmental intestine stricture were found during laparotomy. $d$ Representative histological findings of the resected specimen: ulceration, nonspecific inflammatory infiltrate and granulation tissue formation. 